\title{
Emotional Intelligence and Personality in Anxiety Disorders
}

\author{
Nathalie P. Lizeretti, ${ }^{1,2,3}$ María Vázquez Costa, ${ }^{1,3}$ and Ana Gimeno-Bayón ${ }^{4}$ \\ ${ }^{1}$ Center for Research and Development of Emotional Intelligence (CIDIE), C/Marià Fortuny, 26-28, 1-6a , 08301 Mataró, Spain \\ ${ }^{2}$ FPCEE Blanquerna, Ramon Llull University, C/Císter 34, 08022 Barcelona, Spain \\ ${ }^{3}$ Maresme Health Consortium C/Prolongació Cirera, s/n, 08304 Mataró, Spain \\ ${ }^{4}$ Erich Fromm Institute of Humanist Psychotherapy, C/Madrazo, 113 Entlo. 2 08021 Barcelona, Spain
}

Correspondence should be addressed to Nathalie P. Lizeretti; nathaliepl@blanquerna.url.edu

Received 24 July 2014; Accepted 21 November 2014; Published 16 December 2014

Academic Editor: Takahiro Nemoto

Copyright (C) 2014 Nathalie P. Lizeretti et al. This is an open access article distributed under the Creative Commons Attribution License, which permits unrestricted use, distribution, and reproduction in any medium, provided the original work is properly cited.

\begin{abstract}
Anxiety disorders (AD) are by far the most frequent psychiatric disorders, and according to epidemiologic data their chronicity, comorbidities, and negative prognostic constitute a public health problem. This is why it is necessary to continue exploring the factors which contribute to the incidence, appearance, and maintenance of this set of disorders. The goal of this study has been to analyze the possible relationship between Emotional Intelligence (EI) and personality disorders (PersD) in outpatients suffering from AD. The sample was made up of 146 patients with AD from the Mental Health Center at the Health Consortium of Maresme, who were evaluated with the STAI, MSCEIT, and MCMI-II questionnaires. The main findings indicate that $89,4 \%$ of the patients in the sample met the criteria for the diagnosis of some PersD. The findings also confirm that patients with AD present a low EI, especially because of difficulties in the skills of emotional comprehension and regulation, and the lack of these skills is related to a higher level of anxiety and the presence of PersD. These findings suggest the need to consider emotional skills of EI and personality as central elements for the diagnosis and treatment of $\mathrm{AD}$.
\end{abstract}

\section{Introduction}

1.1. Anxiety Disorders. Due to their high population prevalence, their tendency to become chronic, comorbidities, and bad prognostic, anxiety disorders (AD) constitute an important public health problem [1]. Thus, generalized anxiety disorder (GAD) and substance abuse disorder are the most frequent psychiatric pathologies in the general population [2]. In the specific realm of phobic psychopathology, the rate of lifetime prevalence of panic disorder is around $4 \%$ and agoraphobia is about $2 \%$, and $1 \%$ of the population is diagnosed with panic disorder with agoraphobia (PDA) $[3,4]$. All of these emotional problems linked to high levels of anxiety are frequently complicated due to the presence of medical and psychiatric comorbidities, where depressive disorders and substance abuse from Axis I of DSM-IV are most commonly associated with anxiety disorders [3].

1.2. Personality Disorders. Between $33.9 \%$ and $95 \%$ of patients with anxiety disorder meet the criteria for a personality disorder (PersD) $[3,5,6]$, and $20.5 \%$ of patients meet criteria for more than one personality disorder simultaneously [7]. The most prevalent personality disorders are those in cluster $\mathrm{C}$ (especially Avoidant and Dependent), but it is also frequent to find personality disorders from cluster B (Borderline and Histrionic) and even Narcissist and Antisocial [1,7]. It is less frequent to find cluster A personality disorders associated with anxiety disorders, although they have been found in some research [3].

PersD are a factor of bad prognostic of $\mathrm{AD}$, affecting its clinical course, severity, and dysfunction level. For example, patients with $\mathrm{AD}$ and comorbid PersD have higher levels of anxiety, depression, and agoraphobic symptoms [7]. Some studies explore the relationship between $\mathrm{AD}$ and personality traits (which may not meet the disorder criteria per se) and conclude that even these personality traits are risk predictors for $\mathrm{AD}$, and the more extreme these traits, the higher the level of dysfunction [8].

In order to evaluate PersD, the most commonly used research instrument is the MCMI-II test [9]. In Theodore 
Millon's perspective, personality is understood as an organizing principle which would explain both psychopathology (PersD) and the context to interpret the clinical disorders in Axis I of DSM [9]. According to this model, the comorbidity between Axes I and II is not surprising, and the high prevalence of PersD in clinical samples is explained by the aggravation of nonadaptive behavior patterns when they concur with emotional alterations. However, on some occasions this finding has been interpreted as a tendency of the test instrument to overdiagnose PersD; thus the data must be interpreted carefully [10].

1.3. IE in Anxiety and Personality Disorders. Emotional Intelligence (IE) has been described as a set of skills to identify, facilitate, understand, and regulate emotions, which allows use of emotional knowledge to achieve a higher adjustment and psychological wellbeing [11]. In order to evaluate Emotional Intelligence, the model has developed self-completion scales and a multidimensional measure based on execution tasks, the Mayer-Salovey-Caruso Emotional Intelligence Test V2.0 (MSCEIT) [12] which requires the resolution of problems with emotions. Studies performed on these instruments show that people with a high EI tend to have a more positive frame of mind; they are more able to redress their mood after unpleasant emotions and score lower on anxiety, depression, and stress $[13,14]$. However, given that emotional regulation is crucial for the development and maintenance of adequate mental health [15] there is still insufficient research on the relationship between EI, psychopathological disorders, and PersD.

Recent studies prove that patients with several clinical disorders present deficits in EI, as measured with the MSCEIT skill test [16-19] and with self-assessment measures [20-22]. The studies which explore EI in patients with AD show that the deficit in any of these emotional skills can somehow contribute to the development and maintenance of the AD. A finding is that for patients with social anxiety there is a strong correlation between the gravity of symptoms and the difficulty to adequately perceive emotions and use them to facilitate their thinking [17]. Patients with PDA have significantly lower scores in emotional comprehension and regulation skills than healthy control group participants, which indicates that deficits in emotional comprehension and integration are involved in the phenomenology of panic disorder [19]. Another observation is that patients with GAD pay excessive attention to their emotions and have important difficulties in repairing their negative moods, as well as the fact that the presence and gravity of this disorder's symptoms are related to their incapacity to clearly distinguish between different emotional states. This implies that the perceived incapacity to manage one's emotions could be a vulnerability factor in the development of GAD [20].

The difficulty in managing emotional states is also a key factor in personality disorders. Some pathological personality traits have been associated with important deficits in EI in nonclinical subjects, such as Psychopathic [23], Schizotypal [24], and Borderline $[25,26]$ traits. Thus, we think that the presence of PersD in patients with $\mathrm{AD}$ could be associated with higher deficits in EI. The goal of this research has been to analyze the relationship between EI and PersD in patients with $\mathrm{AD}$, with the initial hypothesis that the presence of PersD will be associated with deficits in emotional skills and a higher severity of $\mathrm{AD}$.

\section{Method}

2.1. Participants. The participants were 153 patients from the Mental Health Center of the Maresme Health Consortium (in Mataró, Spain) diagnosed with AD by the reference psychiatrist or psychologist and referred over to psychotherapy services. The period of data collection was from February 2007 to July 2009. Those who presented a severe psychotic disorder $(n=1)$, mental retardation, literacy difficulties $(n=$ $3)$, or difficulties in understanding the language $(n=3)$ were excluded from the study. None of the patients had an organic brain disorder or a history of substance abuse. The sample was made up of 146 Caucasian patients (84.2\% women), of which 54.8\% $(n=80)$ met DSM-IV-TR diagnostic criteria for PDA, and $45.2 \%(n=66)$ met the diagnostic criteria for GAD. All patients included in the study provided written informed consent. The age of patients was between 18 and 65 years $(M=38.05 ; \mathrm{SD}=10.72)$, and the average time span of disorder progression was 6.77 years $(\mathrm{SD}=5.36)$. The anxiety level of the sample was high $(M=37.73$; $\mathrm{SD}=10.40)$, thus standing within percentiles 95 and 96 , as measured with the STAI-R [27].

2.2. Materials and Procedure. After being diagnosed, the patients who met the inclusion criteria were informed of the research purposes and expressed their informed consent to participate. This research was approved by the ethical review board. The evaluation of participants was preformed through two 90-minute individual interviews (within 10 days apart) prior to psychotherapeutic treatment. The Structured Clinical Interview for Axis I DSM-IV (SCID-I Clinical Version) [28] was used in the first session in order to confirm the diagnosis for Axis I. Millon's Multiaxial Clinical Inventory (MCMI-II) [9] was also administered for the diagnosis of disorders on Axis II of DSM-IV. Throughout the 176 items of dichotomic response, MCMI-II gathers aspects of pathological personality in 26 scales (4 reliability scales, 10 basic personality scales, 3 pathologic personality scales, and 9 clinical syndrome scales). The validity indexes range between 0.71 for major depression scale and 0.85 for the Dependent PersD scale. For the present study, the most conservative criteria were chosen, considering as PersD the scores over TB $>84$ [6].

During the second interview, EI was evaluated using MSCEIT V2.0 [12]. This test has 141 items with five response choices which measure the subject's skills in different emotional tasks. Test questions are grouped in four branches and two fields of construct: the experiential field which includes perception skills and use of emotions to facilitate thinking and the field of reasoning, which includes emotional comprehension and regulation. This test allows us to obtain a total EI coefficient, scores for two fields, and the four branches of skills. The alpha indexes of reliability for the main scales under the general scoring criteria range between 
TABLE 1: Patients with clinical significant punctuations in the personality scales.

\begin{tabular}{|c|c|c|c|c|c|}
\hline & PersD $(>84)$ & PDA & GAD & \multirow{2}{*}{$\chi^{2}$} & \multirow{2}{*}{$P$ value } \\
\hline & $\%(n)$ & $\%(n)$ & $\%(n)$ & & \\
\hline Schizoid & $43.3 \%(61)$ & $40.8 \%(31)$ & $46.2 \%(30)$ & .411 & .319 \\
\hline Avoidant & $22.0 \%(31)$ & $25.0 \%(19)$ & $18.5 \%(12)$ & .873 & .233 \\
\hline Dependent & $48.2 \%(68)$ & $47.4 \%(36)$ & $49.2 \%(32)$ & .049 & .479 \\
\hline Histrionic & $21.3 \%(30)$ & $21.1 \%(16)$ & $21.5 \%(14)$ & .005 & .553 \\
\hline Narcissistic & $24.1 \%(34)$ & $23.7 \%(18)$ & $24.6 \%(16)$ & .017 & .526 \\
\hline Antisocial & $27.7 \%(39)$ & $27.6 \%(21)$ & $27.7 \%(18)$ & .000 & .571 \\
\hline Aggressive-Sadistic & $23.4 \%(33)$ & $18.4 \%(14)$ & $29.2 \%(19)$ & 2.284 & .095 \\
\hline Compulsive & $60.3 \%(85)$ & $52.6 \%(40)$ & $69.2 \%(45)$ & 4.032 & $.033^{*}$ \\
\hline Passive-Aggressive & $14.4 \%(26)$ & $15.8 \%(12)$ & $21.5 \%(14)$ & .770 & .254 \\
\hline Self-Defeating & $28.4 \%(40)$ & $27.6 \%(21)$ & $29.2 \%(19)$ & .044 & .490 \\
\hline Schizotypal & $28.4 \%(40)$ & $27.6 \%(21)$ & $29.2 \%(19)$ & .044 & .490 \\
\hline Borderline & $26.2 \%(37)$ & $25.0 \%(19)$ & $27.7 \%(18)$ & .131 & .432 \\
\hline Paranoid & $34.0 \%(48)$ & $30.3 \%(23)$ & $36.5 \%(25)$ & 1.049 & .199 \\
\hline
\end{tabular}

$\chi^{2}$ significance value; ${ }^{*} P<0.050$.

0.93 for total EI and 0.79 for emotional facilitation. Out of the different scales in the test, age and gender were jointly used. The scores obtained were standardized according to the normative sample $(M=100 ; \mathrm{SD}=15)$. Score ranges for all scales are the following: Improve ( $0-<70)$, Consider developing $(\geq 70-<90)$, Competent $(\geq 90-<110)$, Skilled $(\geq 110-<130)$, and Expert ( $\geq 130)$.

2.3. Analysis. A frequency analysis for PersD was preformed, to prove whether there were differences between patients with PDA and GAD in comparison with the demographic variables, the level of anxiety, and EI in the chi-squared and Student's $t$-test. After this, a covariance analysis was preformed, in order to control the effect of demographic variables on the scores for EI skills and the personality scales. To finish, a Pearson correlation analysis was preformed between the subscales of the MSCEIT and the scales of the MCMI-II. These analyses were carried out with the statistical software package SPSS version 15.0.

\section{Results}

3.1. PersD Frequency Analysis. The frequency of clinically significant scores is high for all the personality scales (see Table 1). The result in the Compulsive scale is extreme, since $60.3 \%$ of the sample exceeds the cutoff point; thus it is found more frequently amongst patients with GAD. The analysis also indicated that $83.7 \%$ of the sample met criteria for more than one PersD, according to the MCMI-II ( $M=4.23$; SD $=2.633$ ), where the presence of two PersD was the most frequent test result (17.1\% of patients).

3.2. Descriptive Statistics. Among the personality scales, apart from the high scores in the Compulsive scale (which exceed the $\mathrm{TB}>84$ ), the scores in the Dependent and
Schizoid scales (above TB > 74) also stand out, indicating an important presence of these PersD amongst the participants (see Table 2). Low EI coefficient scores also appear in the four skills branches, where the greatest difficulties are in the area of reasoning (comprehension and regulation), scoring more than one standard deviation below the standardized mean. Student's $t$-test shows that amongst patients with PDA and GAD there are differences in age, and patients with GAD obtain higher scores in the Aggressive-Sadistic scale $(P=$ 0.028 ) and Compulsive scale $(P=0.044)$, but no significant EI differences were found between both diagnoses.

3.3. Variance Analysis. ANOVA models were thereafter preformed to compare the mean scores of the MCMI-II and MSCEIT between patients with PDA and patients with GAD, checking for possible confounding effects of age, gender, and years of disorder progression. The results indicate a significant positive effect of years of disorder progression on the Phobic scale scores $(F=6.507 ; P=0.012)$ for PDA patients. There is also a significant positive effect of years of disorder progression on the Aggressive-Sadistic scale $(F=$ 4.404; $P=0.038$ ) and on the identification of emotions skills $(F=4.174 ; P=0.043)$ for the GAD patients.

3.4. Correlations. Table 3 shows several statistically significant correlations between personality scales, anxiety scales, and EI skills. Anxiety correlated positively with PersD, except for cluster B and Compulsive patients. Emotional facilitation correlated negatively with the Schizotypal, Dependent, and Self-Defeating scales. Emotional comprehension correlated negatively with Schizoid, Phobic, Self-Defeating, Borderline, and Dependent scales, but it correlated positively with the scales in cluster B (Histrionic, Narcissistic, and Antisocial). The emotional regulation skills also correlated negatively with most of the personality scales. However, emotion identification did not correlate with any of the personality scales, and 


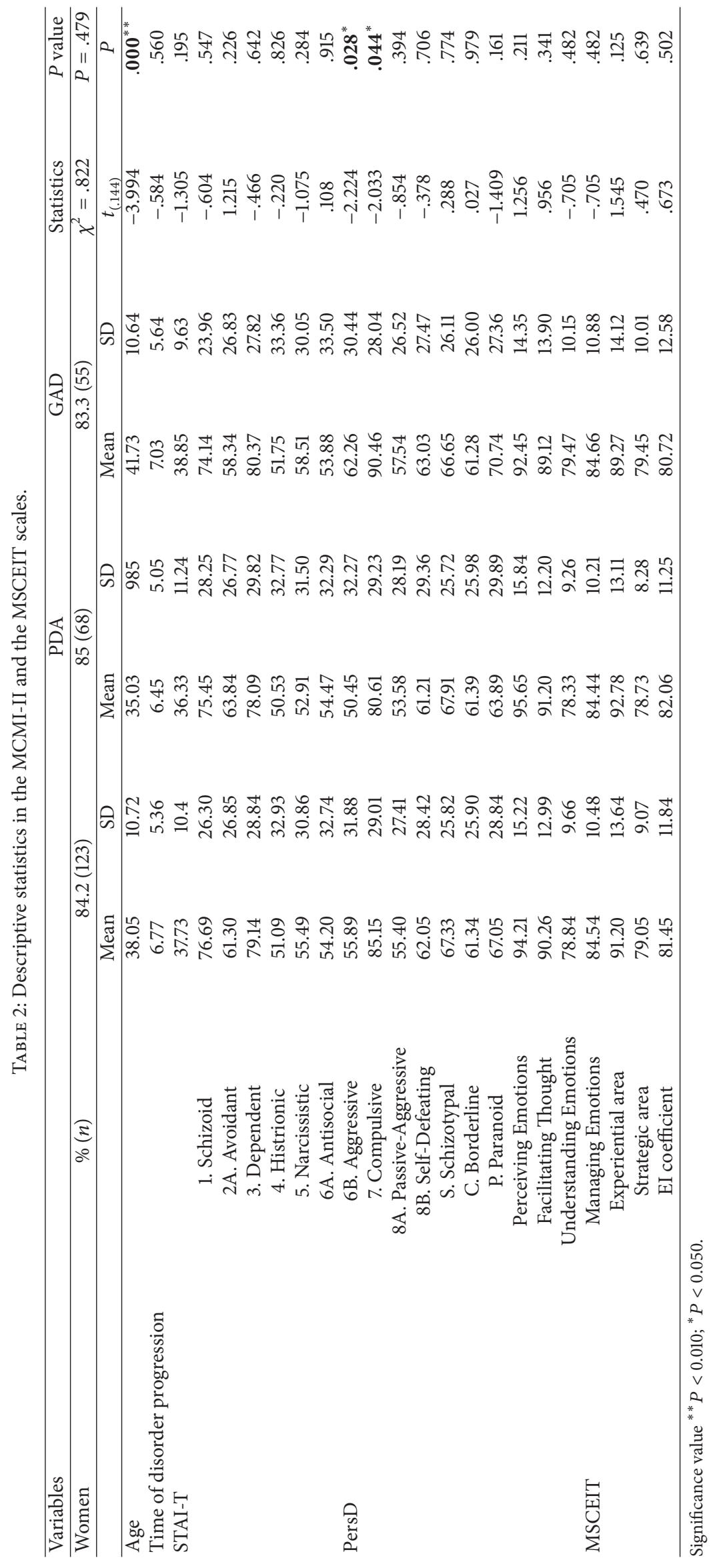


TABLE 3: Correlations between personality, anxiety, and IE's skills.

\begin{tabular}{|c|c|c|c|c|c|c|}
\hline \multirow{2}{*}{$\begin{array}{l}\text { Variables } \\
\text { PersD }\end{array}$} & \multirow{2}{*}{$\begin{array}{c}\text { Anx. } \\
\text { STAI-T }\end{array}$} & \multicolumn{5}{|c|}{ Emotional Intelligence } \\
\hline & & $\begin{array}{c}\text { Perceiving } \\
\text { Emotions }\end{array}$ & $\begin{array}{c}\text { Facilitating } \\
\text { Thought }\end{array}$ & $\begin{array}{l}\text { Understanding } \\
\text { Emotions }\end{array}$ & $\begin{array}{l}\text { Managing } \\
\text { Emotions }\end{array}$ & EI coefficient \\
\hline 1. Schizoid & $.400^{* *}$ & -.116 & -.011 & $-.228^{* *}$ & -.146 & $-.169^{*}$ \\
\hline 2A. Avoidant & $.426^{* *}$ & -.125 & -.086 & $-.236^{* *}$ & $-.220^{* *}$ & $-.228^{* *}$ \\
\hline 3. Dependent & $.335^{* *}$ & -.030 & $-.198^{*}$ & $-.208^{*}$ & $-.282^{* *}$ & $-.261^{* *}$ \\
\hline 4. Histrionic & -.098 & .122 & .000 & $.211^{*}$ & .007 & .126 \\
\hline 5. Narcissistic & -.064 & -.038 & .033 & $.210^{*}$ & .012 & .081 \\
\hline 6A. Antisocial & -.075 & .052 & .061 & $.282^{* *}$ & .060 & .124 \\
\hline 6B. Aggressive & .032 & -.101 & -.037 & .153 & .049 & -.014 \\
\hline 7. Compulsive & .106 & -.014 & -.052 & -.072 & .030 & -.054 \\
\hline 8A. Passive-Aggressive & $.306^{* *}$ & -.067 & -.046 & -.107 & $-.224^{* *}$ & -.147 \\
\hline 8B. Self-Defeating & $.478^{* *}$ & -.076 & $-.208^{*}$ & $-.334^{* *}$ & $-.323^{* *}$ & $-.324^{* *}$ \\
\hline S. Schizotypal & $.452^{* *}$ & -.192 & $-.254^{* *}$ & $-.351^{* *}$ & $-.366^{* *}$ & $-.405^{* *}$ \\
\hline C. Borderline & $.387^{* *}$ & -.087 & -.165 & $-.317^{* *}$ & $-.307^{* *}$ & $-.292^{* *}$ \\
\hline P. Paranoid & $.203^{* *}$ & -.147 & -.120 & -.028 & $-.200^{*}$ & $-.192^{*}$ \\
\hline
\end{tabular}

Significance value ${ }^{* *} P<0.010 ;{ }^{*} P<0.050$.

none of the EI skills correlated with the Aggressive-Sadistic scale and Compulsive scale.

\section{Discussion and Conclusions}

No studies were found that investigated the relationship between EI with the skills model and PersD in patients with psychopathology. In response to the need for research in this realm, the goal of the present research has been to analyze the relationship between EI and PersD for patients with AD. Our hypothesis stated that the patients with $\mathrm{AD}$ would present difficulties managing their emotions, which would translate into a low level of EI, and that this would be related to more anxiety and the presence of PersD.

The results indicate that $89.4 \%$ of the patients in the sample met the criteria for the diagnosis of some PersD (according to the MCMI-II). This data is superior to that found in some previous studies, which found a frequency of PersD approximately at 33.9\% [7] and 46\% [5], but it is similar to some other studies that found that up to $95 \%$ of patients with $\mathrm{AD}$ meet criteria for a PersD, and $20.5 \%$ meet criteria for more than one $[3,6]$.

The most prevalent PersD were Compulsive, Dependent, and Schizoid. The high prevalence of these PersD has also been noted by numerous researches [7], but the prevalence of Schizoid PersD has been higher in this case than in other studies $[3,29]$. The Schizoid PersD could be related to the severity of the $\mathrm{AD}$, being more frequent amongst patients with a severe PDA. Avoidance and progressive isolation to which it leads could accentuate these personality traits.

In terms of prevalence, the only significant differences found between PDA and GAD were in the Compulsive scale. Previous studies have found higher rates of obsessivecompulsive personality disorder (OCPD) in patients with GAD than in patients with PDA [30].
Our results also confirm that patients with $\mathrm{AD}$ present a low EI, without finding significant differences between PDA and GAD. Specifically, patients show sufficient competence in identification and facilitation skills but have difficulties in the skills of emotional comprehension and regulation (which imply greater processing complexity). These results are consistent with the studies performed within the Emotional Intelligence paradigm $[17,19,20]$ and outside of this paradigm [31], which show that in $\mathrm{AD}$ the symptoms are associated with a higher emotional intensity, low emotional comprehension capacity, negative reactions towards one's own emotions, and difficulties in emotional management.

Also, we have observed that the years of $\mathrm{AD}$ progression have a significant impact on personality and EI, indicating that chronicity of the disorder is related with lower emotional management skills. The longer the time of disorder progression is, the more the Phobic personality traits are accentuated in PDA and the Aggressive-Sadistic traits are accentuated in GDA, decreasing the emotional identification skills. These data could lead to two complementary explanatory hypotheses. The first hypothesis is that baseline PersD is a vulnerability factor [9], which increases the severity and risk of chronicity of the $\mathrm{AD}$ [7]. The second hypothesis is that anxiety symptoms experienced for a long time can modify personality structure. This hypothesis offers a circular view of the interaction between disorders in Axis I and Axis II for the patients with $\mathrm{AD}$ and suggests the need to contemplate personality in the evaluation and treatment of $\mathrm{AD}$.

In accordance with our hypothesis, the correlation analysis indicates that the presence of PersD is associated with a higher anxiety and a lower EI. Among the most prevalent ones, the Dependent PersD is related to difficulties in the use of emotion to facilitate thinking but, especially in the comprehension and regulation of emotional experiences. The Dependent person tends to disconnect her own emotions, 
and her thought and behavior are guided by significant others (on whom she depends) rather than her own emotional needs. For the Schizoid PersD it is unsurprising to find an association with a high anxiety and a difficulty in understanding emotional states in self and others. It is surprising to find a total absence of correlation between obsessivecompulsive PersD and EI. Possibly this lack of correlation could be explained due to the rationalizing tendency of this OCPD, which acts as a control mechanism at the expense of spontaneity and authenticity of emotional experience.

It is also interesting to find a positive relation between the Histrionic, Narcissistic, and Antisocial scales with emotional comprehension, although it is true that these personality types show a certain emotional dominance through their manipulative and seductive behaviors. The pioneers of the EI concept, Salovey and Mayer [32], already warned that "those whose [emotional] skills are channeled antisocially may create manipulative scenes or lead others sociopathically to nefarious ends" (p.198). So, emotion-regulation knowledge "has a dark side as well"; that is, it facilitates both prosocial and interpersonally deviant behavior by enhancing the motivational effects of traits [33]. In a person with Narcissistic or Antisocial traits (which imply an egocentric attitude of despising others' needs and social norms), a high emotional comprehension could be a powerful manipulation weapon. For example, it has been noted that the school bullies scored higher on emotion understanding than did nonbullies [34].

Referring to these results and keeping in mind the intrinsic goodness of authentic emotions (in their instinctive and adaptive character) [35] one may wonder whether the emotional skills assessed with the MSCEIT are really referring to an authentic, natural, and deep EI or, on the contrary, they refer to a set of emotional manipulation skills or manipulative EI. In any case, it is necessary to discriminate between one and the other type of EI, and for this matter personality is a key factor to keep in mind.

In this research, we have analyzed the relationship between different dimensions of EI and PersD in patients with AD. Our results offer two possible hypotheses which could occur simultaneously: first, that deficits in EI skills are an important factor in the etiology of personality disorders and, second, that the pathological personality traits tamper the development of emotional skills, which could partly explain the incidence of these clinical disorders in Axis I. In any case, it seems obvious that the patients with $\mathrm{AD}$ need to develop their emotional reasoning skills to attain an adequate emotional competence, and this suggests that interventions aimed at the development of these emotional skills can contribute to a higher efficacy of the current treatments. However, more research will be necessary to clarify the relationship between EI and the different PersD in clinical patients.

\section{Limitations and Future Research Directions}

One of the strong points of this study is the use of the MSCEIT skill test to measure IE. Most studies on EI discussing clinical issues rely on self-report measures. Our study also presents some limitations such as the reduced sample size or the exclusive use of MCMI-II for the diagnosis of PersD. On the other hand, the cross-sectional study design does not allow establishing causal relationship between the observed associations. Further longitudinal research is needed to clarify causal relation between deficits in EI and PersD in AD patients.

\section{Highlights}

We highlight the following points: (i) almost $90 \%$ of patients suffering AD present a Compulsive, Dependent, or Schizoid PersD, (ii) patients with AD present an EI level which is lower than the general population, (iii) patients with AD have greater difficulties in emotional comprehension and regulation, and (iv) suffering an $\mathrm{AD}$ together with a PersD indicates that fewer emotional management skills are available.

\section{Conflict of Interests}

The authors declare that there is no conflict of interests regarding the publication of this paper.

\section{References}

[1] B. L. Milrod, A. C. Leon, J. P. Barber, J. C. Markowitz, and E. Graf, "Do comorbid personality disorders moderate panicfocused psychotherapy? An exploratory examination of the American psychiatric association practice guideline," The Journal of Clinical Psychiatry, vol. 68, no. 6, pp. 885-891, 2007.

[2] J. Alonso, M. C. Angermeyer, S. Bernert et al., "Prevalence of mental disorders in Europe: results from the European Study of the Epidemiology of Mental Disorders (ESEMeD) project," Acta Psychiatrica Scandinavica, Supplement, vol. 109, no. 420, pp. 2127, 2004.

[3] B. F. Grant, D. S. Hasin, F. S. Stinson et al., "The epidemiology of DSM-IV panic disorder and agoraphobia in the United States: results from the National epidemiologic survey on alcohol and related conditions," The Journal of Clinical Psychiatry, vol. 67, no. 3, pp. 363-374, 2006.

[4] R. C. Kessler, A. M. Ruscio, K. Shear, and H.-U. Wittchen, "Epidemiology of anxiety disorders," Current Topics in Behavioral Neurosciences, vol. 2, pp. 21-35, 2010.

[5] R. C. Durham, T. Murphy, T. Allan, K. Richard, L. R. Treliving, and G. W. Fenton, "Cognitive therapy, analytic psychotherapy and anxiety management training for generalised anxiety disorder," The British Journal of Psychiatry, vol. 165, pp. 315-323, 1994.

[6] S. Wetzler, R. S. Kahn, W. Cahn, H. M. van Praag, and G. M. Asnis, "Psychological test characteristics of depressed and panic patients," Psychiatry Research, vol. 31, no. 2, pp. 179-192, 1990.

[7] M. Ozkan and A. Altindag, "Comorbid personality disorders in subjects with panic disorder: do personality disorders increase clinical severity?" Comprehensive Psychiatry, vol. 46, no. 1, pp. 20-26, 2005.

[8] M. Brandes and O. J. Bienvenu, "Personality and anxiety disorders," Current Psychiatry Reports, vol. 8, no. 4, pp. 263-269, 2006.

[9] T. Millon, Millon Multiaxial Clinical Inventory II (MCMI-II), TEA, Madrid, Spain, 1987, Spanish adaptation by Ávila-Espada, 1997. 
[10] F. M. Martín, A. J. Cangas, and M. E. Pozo, "Personality disorders in patients with eating disorders," Psicothema, vol. 21, pp. 33-38, 2009.

[11] J. D. Mayer and P. Salovey, "What is emotional intelligence?" in Emotional Development and Emotional Intelligence Educational Implications, P. Salovey and D. J. Sluyter, Eds., pp. 3-31, Basic Books, New York, NY, USA, 1997.

[12] J. D. Mayer, P. Salovey, D. Caruso, and G. Sitarenios, MayerSalove -Caruso Emotional Intelligence Test (MSCEIT) User's Manual, MHS, Ontario, Canada, 2005.

[13] P. Fernández-Berrocal and N. Extremera, "Emotional intelligence and emotional reactivity and recovery in laboratory context," Psicothema, vol. 18, no. 1, pp. 72-78, 2006.

[14] P. Salovey, L. R. Stroud, A. Woolery, and E. S. Epel, "Perceived emotional intelligence, stress reactivity, and symptom reports: further explorations using the trait meta-mood scale," Psychology and Health, vol. 17, no. 5, pp. 611-627, 2002.

[15] A. Aldao and S. Nolen-Hoeksema, "Specificity of cognitive emotion regulation strategies: a transdiagnostic examination," Behaviour Research and Therapy, vol. 48, no. 10, pp. 974-983, 2010.

[16] J. Hertel, A. Schütz, and C. H. Lammers, "Emotional intelligence and mental disorder," Journal of Clinical Psychology, vol. 65, no. 9, pp. 942-954, 2009.

[17] M. Jacobs, J. Snow, M. Geraci et al., "Association between level of emotional intelligence and severity of anxiety in generalized social phobia," Journal of Anxiety Disorders, vol. 22, no. 8, pp. 1487-1495, 2008.

[18] S. Kee, P. Horan, P. Salovey et al., "Emotional intelligence in schizophrenia," Schizophrenia Research, vol. 107, no. 1, pp. 61-68, 2009.

[19] G. Perna, R. Menotti, G. Borriello, P. Cavedini, L. Bellodi, and D. Caldirola, "Emotional intelligence in panic disorder," Rivista di Psichiatria, vol. 45, no. 5, pp. 320-325, 2010.

[20] N. P. Lizeretti and N. Extremera, "Emotional intelligence and clinical symptoms in outpatients with Generalized Anxiety Disorder (GAD)," Psychiatric Quarterly, vol. 82, no. 3, pp. 253260, 2011.

[21] N. P. Lizeretti, N. Extremera, and A. Rodríguez, "Perceived emotional intelligence and clinical symptoms in outpatiens with mental disorders," Psychiatry Quaterly, vol. 83, pp. 407-418, 2012.

[22] N. P. Lizeretti and A. Rodríguez, "Perceived emotional intelligence in patients diagnosed with panic disorder with agoraphobia," Anxiety and Stress, vol. 18, no. 1, pp. 43-53, 2012.

[23] M. B. Malterer, S. J. Glass, and J. P. Newman, "Psychopathy and trait emotional intelligence," Personality and Individual Differences, vol. 44, no. 3, pp. 735-745, 2008.

[24] F. Aguirre, M. J. Sergi, and C. A. Levy, "Emotional intelligence and social functioning in persons with schizotypy," Schizophrenia Research, vol. 104, no. 1-3, pp. 255-264, 2008.

[25] K. Gardner and P. Qualter, "Emotional intelligence and Borderline personality disorder," Personality and Individual Differences, vol. 47, no. 2, pp. 94-98, 2009.

[26] T. L. Leible and W. E. Snell Jr., "Borderline personality disorder and multiple aspects of emotional intelligence," Personality and Individual Differences, vol. 37, no. 2, pp. 393-404, 2004.

[27] C. D. Spielberger, R. L. Gorsuch, and R. F. Lushene, State-Trait Anxiety Inventory, TEA, Madrid, Spain, 1970.
[28] M. B. First, R. L. Spitzer, M. Gibbon, and J. B. Williams, Structured Clinical Interview for DSM-IV Axis I disorders (SCIDI) Clinical Version, Masson, Barcelona, Spain, 1997, Spanish adaptation by Blanch and Andreu 1999.

[29] I. R. Dyck, K. A. Phillips, M. G. Warshaw et al., "Patterns of personality pathology in patients with generalized anxiety disorder, panic disorder with and without agoraphobia, and social phobia," Journal of Personality Disorders, vol. 15, no. 1, pp. 60-71, 2001.

[30] J. E. Grant, M. E. Mooney, and M. G. Kushner, "Prevalence, correlates, and comorbidity of DSM-IV obsessive-compulsive personality disorder: results from the National Epidemiologic Survey on Alcohol and Related Conditions," Journal of Psychiatric Research, vol. 46, no. 4, pp. 469-475, 2012.

[31] D. S. Mennin, R. G. Heimberg, C. L. Turk, and D. M. Fresco, "Preliminary evidence for an emotion dysregulation model of generalized anxiety disorder," Behaviour Research and Therapy, vol. 43, no. 10, pp. 1281-1310, 2005.

[32] P. Salovey and J. D. Mayer, "Emotional intelligence," Imagination, Cognition and Personality, vol. 9, pp. 185-211, 1990.

[33] S. Côté, K. A. DeCelles, J. M. McCarthy, G. A. van Kleef, and I. Hideg, "The jekyll and hyde of emotional intelligence: emotion-regulation knowledge facilitates both prosocial and interpersonally deviant behavior," Psychological Science, vol. 22, no. 8, pp. 1073-1080, 2011.

[34] J. Sutton, P. K. Smith, and J. Swettenham, "Social cognition and bullying: social inadequacy or skilled manipulation?" British Journal of Developmental Psychology, vol. 17, no. 3, pp. 435-450, 1999.

[35] N. P. Lizeretti, Emotional Intelligence Based Therapy. Treatment Manual, Milenio, Lérida, Spain, 2012. 


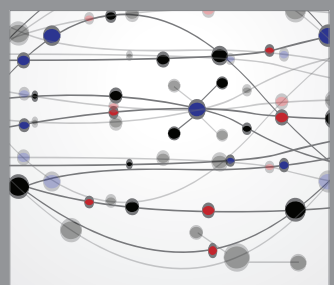

The Scientific World Journal
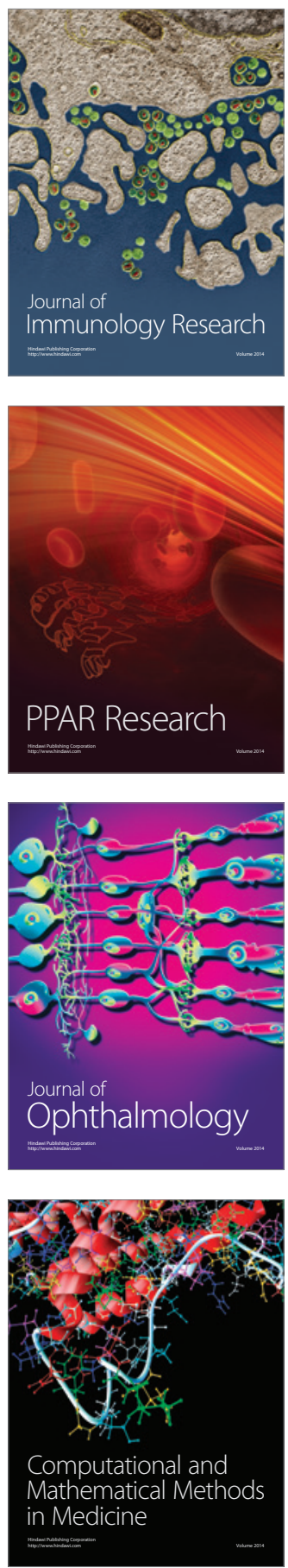

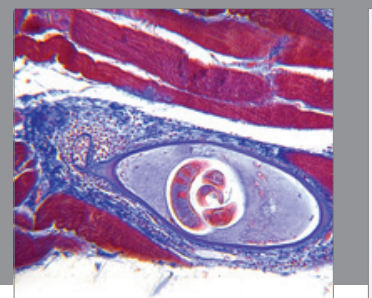

Gastroenterology

Research and Practice
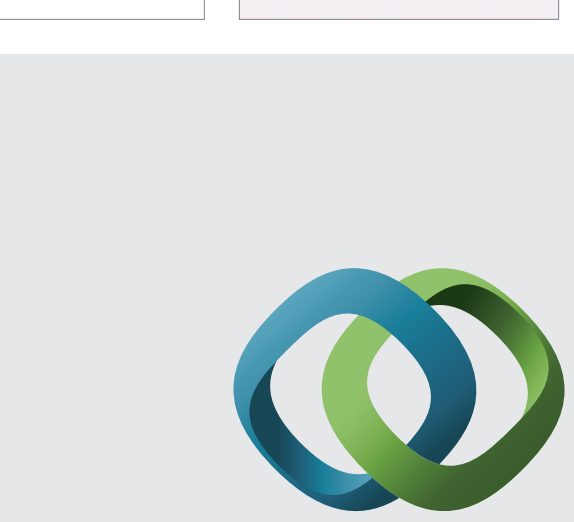

\section{Hindawi}

Submit your manuscripts at

http://www.hindawi.com
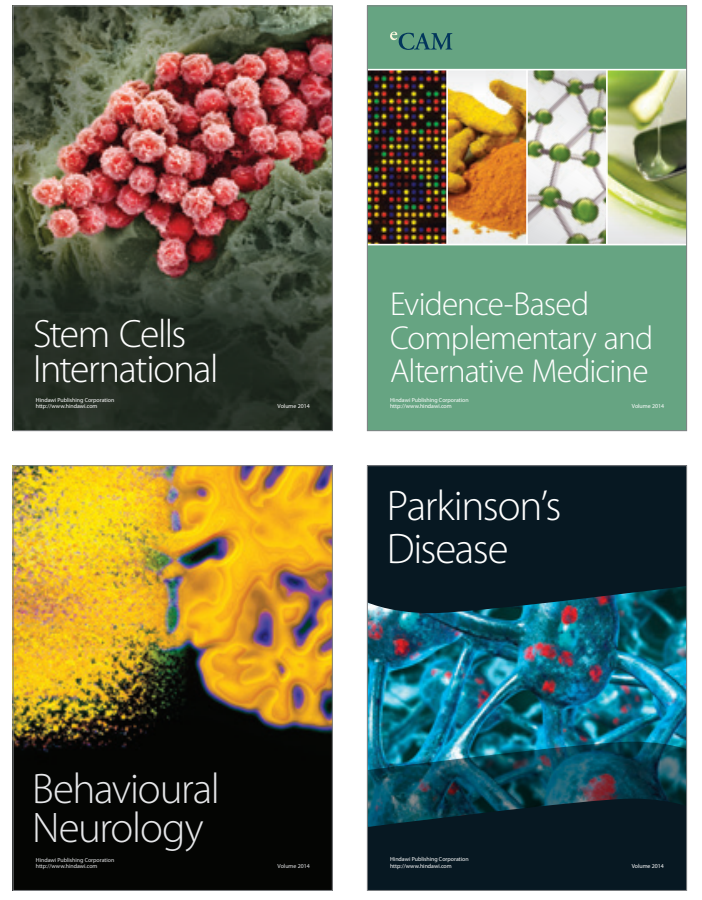
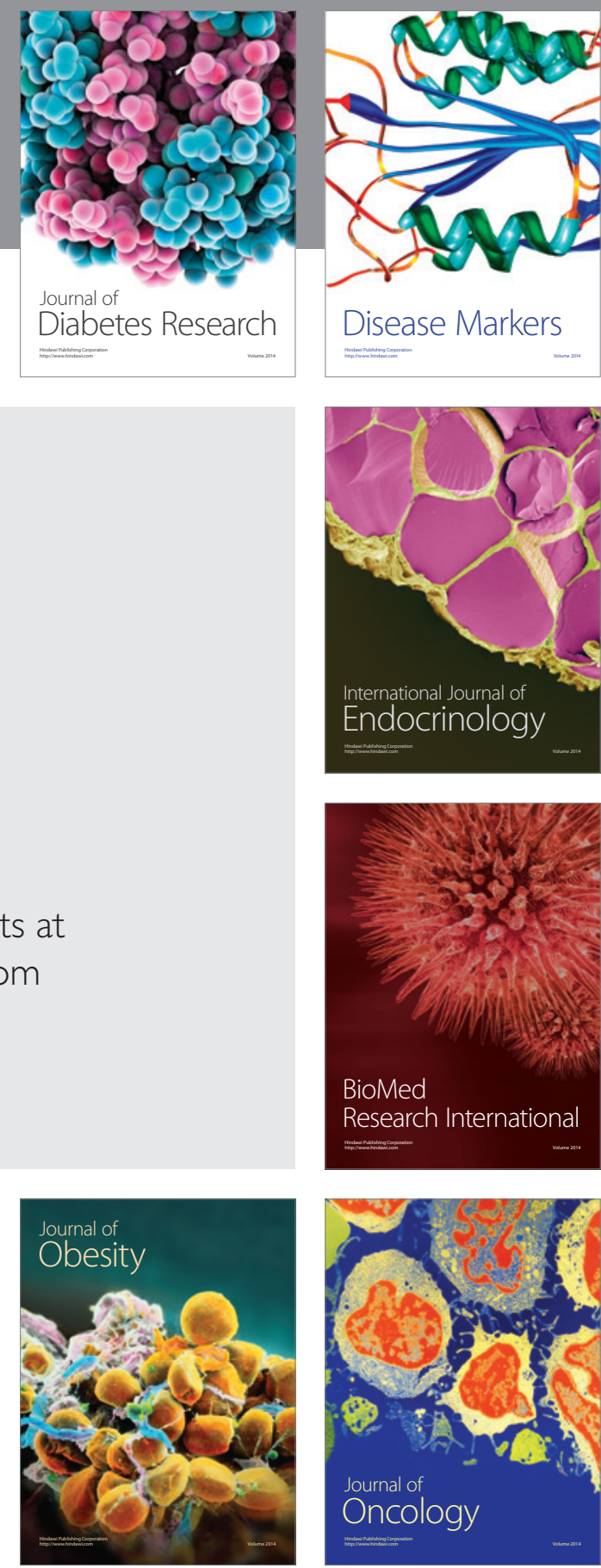

Disease Markers
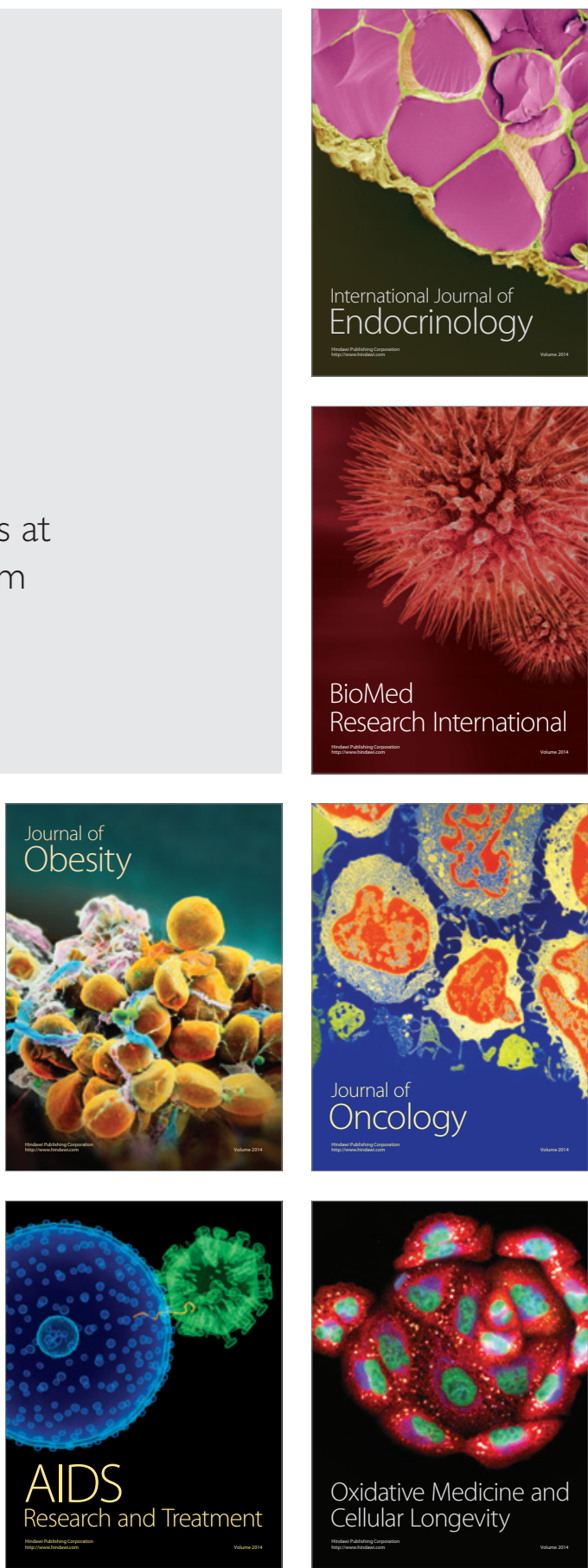